\title{
Micromagnetic Simulation of Voltage-Induced Spin Wave Resonance Properties in Ferromagnetic Nanowires with Perpendicular Anisotropy
}

\author{
X. Ya, R. Imai, T. Tanaka and K. Matsuyama \\ Faculty of Information Science and Electrical Engineering, Kyushu Univ., Motooka 744, Nishi-ku, Fukuoka 819-0395, Japan
}

\begin{abstract}
A fundamental issue in developing spin wave (SW) devices with ultralow power consumption is exploring energy-efficient excitation with nanoscale spatial resolution. We numerically studied the voltage-induced excitation of a geometrically confined standing spin wave (SSW), which is suitable for the downsizing of various SW devices. The micromagnetic configuration of the excited SSW, the resonance spectrum, and the structural size effects were investigated. In addition, a possible application to a SSW-based logic operation was demonstrated in a nanowire with lateral dimensions of $20 \mathrm{~nm} \times 100 \mathrm{~nm}$.
\end{abstract}

Key words: spin wave, ferromagnetic resonance, magnetic anisotropy, micromagnetic simulation

\section{Introduction}

In solid-state physics, spin dynamics in magnetic nanostructures is an active research field ${ }^{1-3}$. In particular, the collective and coherent magnetization precession of spin waves (SWs) has attracted intense research interest ${ }^{4,5}$. The promising abilities of SWs have resulted in the novel research field of so-called magnonics ${ }^{6-8)}$, where the phase or amplitude of a propagating SW is used as a novel information carrier or a signal-processing medium. Energy-efficient excitation or manipulation of SWs is essential to exploit the potential ability of SW-based devices. Two different approaches to this problem, parametric pumping (PP)1) and voltage-controlled magnetic anisotropy (VCMA)9), have been intensively studied over the past years. PP is based on the nonlinear aspect of the spin dynamics, resulting in the excellent frequency-resolved excitation of short wavelength SWs, as experimentally observed in ferromagnetic oxide yttrium iron garnet ${ }^{10-12)}$ and metallic thin films ${ }^{13-19)}$. Voltage-induced manipulations of magnetic properties have also been attracting intense research interest ${ }^{20-24)}$. The superior performance of VCMA has been experimentally confirmed as being an ohmic dissipation free excitation of the ferromagnetic resonance ${ }^{25-27)}$, as opposed to conventional Oersted fields. In particular, the cooperative performance of $\mathrm{PP}$ and VCMA can be effectively exerted in a material system with perpendicular magnetic anisotropy ${ }^{28}$. Recently, the parametric excitation of propagating SWs via VCMA has been investigated using both theoretical ${ }^{29}$ and numerical $^{30}$ approaches. However, the long distance propagation of SWs is still a challenging issue. In this study, the possibility of spatially resolved excitation of geometrically confined standing spin waves (SSWs) in magnetic nanostructures is addressed. A fundamental SW-based logic operation and its design considerations are also studied in the context of an application.

\section{Numerical Model}

Figure 1(a) shows a schematic figure of the investigated device structure, which consists of a perpendicularly magnetized nanowire and an overlaid gate electrode to apply electric fields for the local modulation of the perpendicular magnetic anisotropy (PMA). The following structural and magnetic parameters were adopted in the present study assuming ferromagnetic nanowires with interface-induced perpendicular anisotropy, such as the $\mathrm{CoFeB} / \mathrm{MgO}$ system: the film thickness was $t=0.8 \mathrm{~nm}$, the saturation magnetization was $M_{\mathrm{s}}=1600 \mathrm{emu} / \mathrm{cm}^{3}$, the exchange stiffness constant was $A=1.0 \mu \mathrm{erg} / \mathrm{cm}$, the effective uniaxial perpendicular anisotropy field was $H_{\mathrm{k}}$ $=20 \mathrm{kOe}$, the gyromagnetic ratio was $\gamma=1.76 \times 10^{7}$ $\mathrm{rad} / \mathrm{s} \cdot$ Oe, and the Gilbert damping constant was $\alpha=$ 0.01. The electric field-induced modulation of the PMA was numerically modeled by the local sinusoidal $H_{\mathrm{k}}$ change using the amplitude of $\Delta H_{\mathrm{k}}$ under the gate electrode. The assumed maximum value of $\Delta H_{\mathrm{k}}(500 \mathrm{Oe})$ corresponds to an areal anisotropy energy change of $32.5 \mu \mathrm{J} / \mathrm{m}^{2}$, which is comparable to the experimentally reported values of several tens of $\mu \mathrm{J} / \mathrm{m}^{2}{ }^{24,31,32)}$ The dynamics of the SW caused by the electric field-induced PMA modulation was simulated by numerically integrating the Landau-Lifshitz-Gilbert equation. Subtle thermal agitation as a parallel PP seed was introduced via effective Langevin fields ${ }^{33)}$. The Langevin fields with an amplitude based on fluctuation dissipation theorem and random orientation were applied to micromagnetic numerical cells at each time step $^{34)}$. The fourth-order Runge-Kutta method was adopted as a numerical scheme. The magnetization was assumed to be uniform along the film thickness and the wire width direction, taking into consideration that the dominant magnetization change was along the long axis of the nanowire. Therefore, the nanowire was discretized into a one-dimensional dipole array with a separation of $1 \mathrm{~nm}$, and the dipole coupling was computed using an apparent surface charge model. A 
(a)

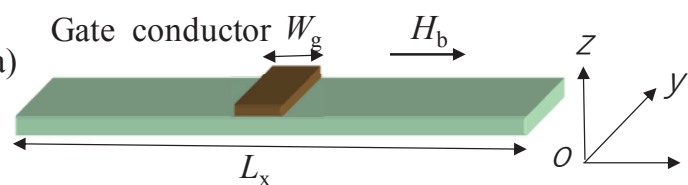

Ferromagnetic nanowire
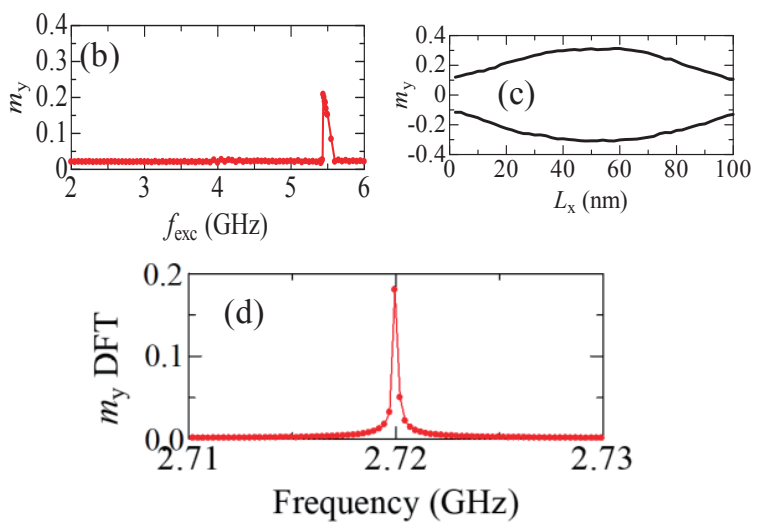

Fig. 1 (a) Schematic of a ferromagnetic nanowire with an overlaid gate electrode for SSW excitation, (b) dependence of the precession amplitude on the excitation frequency, (c) micromagnetic configuration of the SSW, and (d) the frequency spectra of the magnetization precession under the gate electrode.

force-free boundary condition was assumed at the wire edge. The spatial discretization and the time step $\left(10^{-15}\right.$ s) were preliminarily optimized via the micromagnetic standard problem No. $4 .{ }^{35)}$

\section{Results and Discussion}

Figure 1(b) presents the precession amplitude of the magnetization excited with a sinusoidal modulation of the perpendicular anisotropy field $\left(\Delta H_{\mathrm{k}}=500 \mathrm{Oe}\right)$ under a gate electrode with a width of $W_{\mathrm{g}}=10 \mathrm{~nm}$, located at the center of a wire with lateral dimensions of $20 \mathrm{~nm} \times$ $100 \mathrm{~nm}$. The in-plane component of the normalized magnetization $m_{\mathrm{y}}$ at the wire center is plotted as a function of the excitation frequency, $f_{\text {exc. The precession }}$ amplitude sharply decays below the resonance region $\left(f_{\text {exc }}<5.43 \mathrm{GHz}\right)$ while gradually decreasing above the resonance region. The resonance frequency, $f_{\text {res }}$ of 5.44 $\mathrm{GHz}$, is twice the excited precession frequency, as shown in the frequency spectra of the magnetization precession (Fig. 1(d)), calculated from the digital Fourier transform of $m_{\mathrm{y}}$. The micromagnetic configuration of the magnetization precession exhibits a first-order SSW along the long axis of the wire, as shown in Fig. 1(c). These results confirm that the additional in-plane shape anisotropy of ferromagnetic wires with a nanoscale finite length causes an anisotropic precession trajectory accompanied by a time-varying $m_{\mathrm{z}}$ component with a frequency that is twice the precession frequency, which is essential as a seed for parallel parametric excitation. The results also reveal that the parametrically excited SSW exhibits an
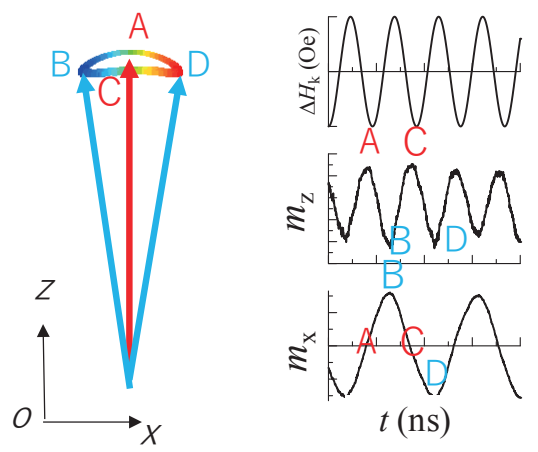

Fig. 2 Elliptic precession trajectory and phase relationship between the sinusoidal perpendicular anisotropy modulation and the magnetization components.

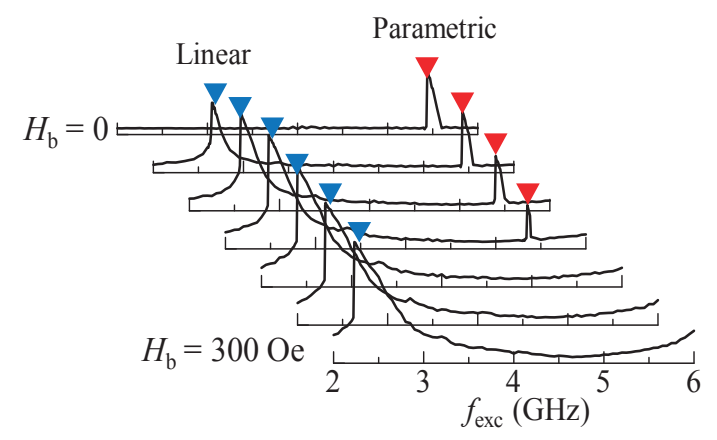

Fig. 3 SSW resonance properties at various external fields $H_{\mathrm{b}}$ applied along the long axis of the wire.

asymmetric resonance profile, similar to the case of a propagating $\mathrm{SW}^{30}$. The relative phase relationship between the time-varying PMA, $m_{\mathrm{x}}$, and $m_{\mathrm{z}}$ indicates an apparent negative susceptibility, as shown in Fig. 2. That is, the increase in the perpendicular anisotropy field $H_{\mathrm{k}}$ leads to a decrease in $m_{\mathrm{z}}$, and vice versa, reflecting the coherent SW resonance behavior.

The external bias field $H_{\mathrm{b}}$ applied along the long axis of the wire causes an additional linear mode excitation of the SSW, where the precession frequency is the same as the excitation frequency, as shown in Fig. 3. Conversely, the parametric mode disappears for $H_{b}$ larger than 200 Oe. It can be reasoned that the precession axis is inclined to the $\mathrm{x}$-axis due to the application of $H_{b}$, which results in the disappearance of the double period change in $m_{z}$ necessary for a parallel parametric excitation seed. As can be seen in Fig. 3, the resonance profile of the parametric mode is markedly narrower than that of the linear mode, which would be suitable for frequency-selective SW-based device operations. Figure 4 compares the dependence of the resonance precession amplitude $m_{\mathrm{y}}$ on the PMA modulation amplitude $\Delta H_{\mathrm{k}}$ for the parametric and linear excitation modes at $H_{\mathrm{b}}=100$ Oe. $m_{\mathrm{y}}$ for the linear mode 


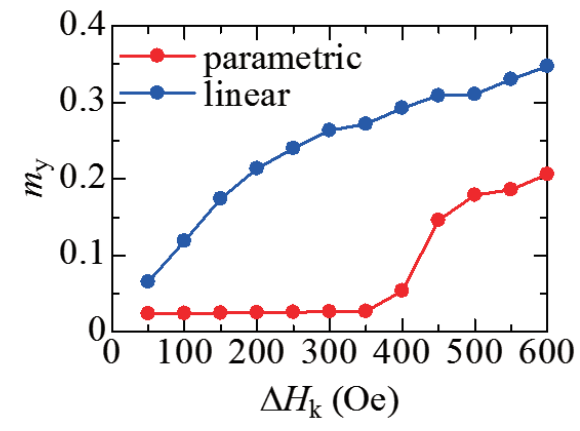

Fig. 4 SSW precession amplitude as a function of the sinusoidal anisotropy field amplitude $\Delta H_{\mathrm{k}}$ for parametric and linear excitations.

linearly increases with increasing $\Delta H_{\mathrm{k}}$ up to approximately 150 Oe and deviates from a linear response for larger $\Delta H_{\mathrm{k}}$. Conversely, the precession amplitude of the SSW in the parametric mode was found to be insensitive to the PMA modulation intensity for $\Delta H_{\mathrm{k}}$ smaller than 350 Oe, resulting in mostly constant values of $m_{\mathrm{y}}$ due to the thermal disturbance. The observed threshold for triggering the parallel parametric excitation has been theoretically predicted for a propagating $\mathrm{SW}^{29,30}$.

The dependences of the parametric excitation properties on representative structural parameters are presented in Figs. 5 and 6. Figure 5(a) compares the resonance profile of SSWs excited in wires with various wire lengths $L_{x}$ and a fixed gate electrode width $\left(W_{\mathrm{g}}=\right.$
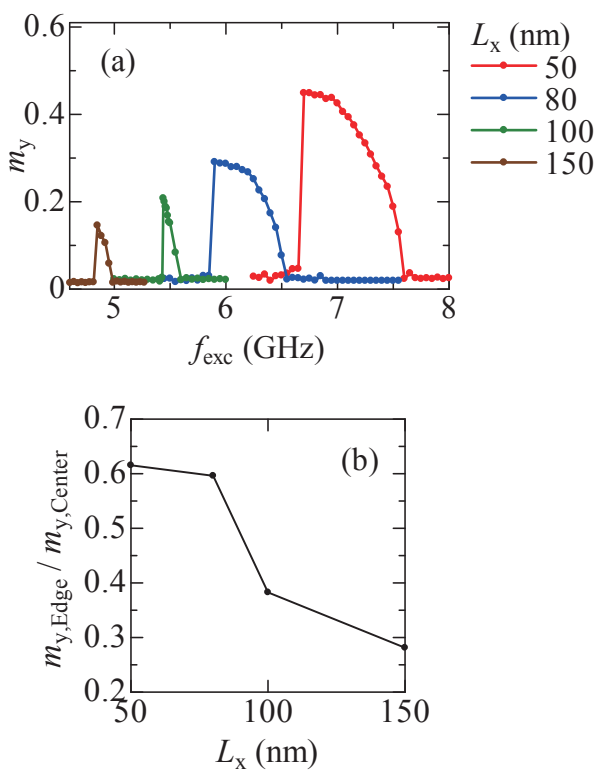

Fig. 5 Dependence of the SSW properties on the wire length $L_{\mathrm{x}}:(\mathrm{a})$ the resonance spectra and (b) the nonuniformity of the precession amplitude along the wire.
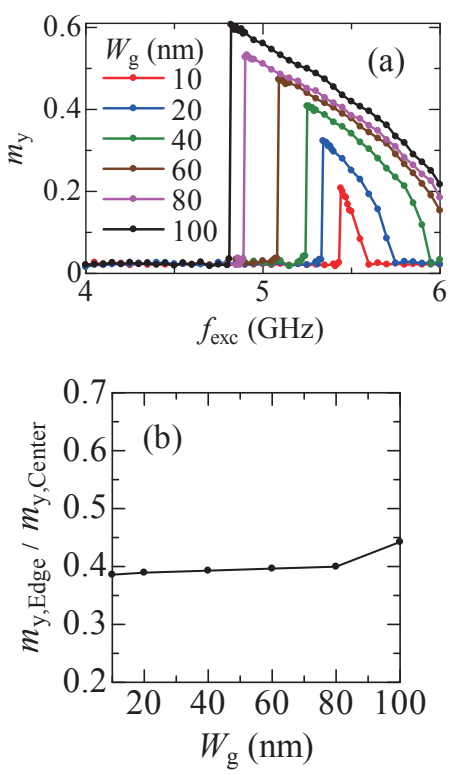

Fig. 6 Dependence of the SSW properties on the gate electrode width wire length $W_{\mathrm{g}}$ : (a) the resonance spectra and (b) the nonuniformity of the precession amplitude along the wire.

$10 \mathrm{~nm}$ ). The values of $f_{\mathrm{opt}}$, at which the precession amplitude reaches its maximum value, increase from $4.8 \mathrm{GHz}$ to $6.7 \mathrm{GHz}$ with $L_{\mathrm{x}}$ decreasing from $150 \mathrm{~nm}$ to $50 \mathrm{~nm}$, reflecting the dispersion relation between the SW length and the excitation frequency. The observed broad spectrum in the short length wire can be associated with the large amplitude precession, geometrically confined in the narrow region; $m_{\mathrm{y}}=0.45$ $\left(L_{\mathrm{x}}=50 \mathrm{~nm}\right), m_{\mathrm{y}}=0.16\left(L_{\mathrm{x}}=150 \mathrm{~nm}\right)$. Figure 5(b) shows the relative ratio of the precession amplitude at the center $\left(m_{y, \text { center }}\right)$ and edge $\left(m_{y, \text { edge }}\right)$ of the wire. The variation of the precession amplitude along the wire axis markedly decreases with decreasing $L_{x}$ and closes to the ferromagnetic resonance mode.

Even though $f_{\text {opt }}$ and the nonuniformity of the precession amplitude also depend on the gate electrode width $W_{\mathrm{g}}$, as shown in Fig. 6(a), (b), this dependency is relatively weak compared to that on the wire length. That is, the increase in the values of $W_{\mathrm{g}}$, which ranged from $10 \mathrm{~nm}$ to $100 \mathrm{~nm}$, resulted in $15 \%$ and $18 \%$ increases in $f_{\text {opt }}$ and $m_{y, \text { edge }} / m_{y, \text { center }}$, respectively. Figure 6(a) shows that the precession amplitude monotonously increases with increasing $W_{\mathrm{g}}$. These results indicate a spatial integration effect in the voltage-induced excitation of the SSW, which can be applied to a logic operation as follows.

Figure 7(b) presents the frequency spectra of the precession amplitude excited with two electrodes located $25 \mathrm{~nm}$ and $75 \mathrm{~nm}$ from the wire end (Fig. 7(a)). The values of $L_{\mathrm{x}}$ and $W_{\mathrm{g}}$ were chosen to be $100 \mathrm{~nm}$ and 10 $\mathrm{nm}$, respectively. A bias field of 100 Oe was applied along the wire axis. The two plots show the results for the different phase differences $\Delta \phi(=0, \pi)$ between the sinusoidal anisotropy changes in the two electrodes. 

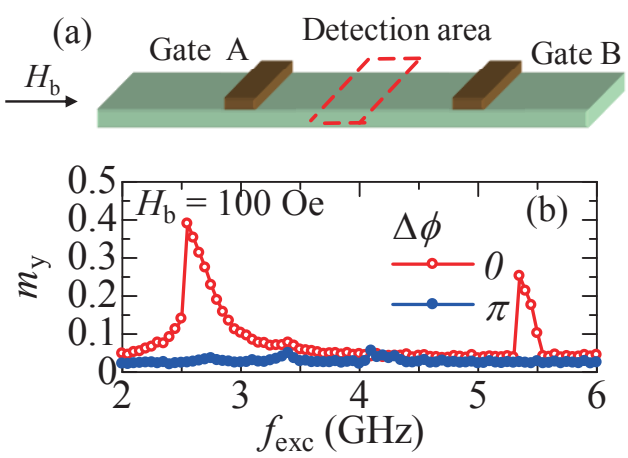

Fig. 7 (a) Schematic of two-generator device. (b) Comparison of the frequency spectra between the in-phase $(\Delta \phi=0)$ and opposite-phase $(\Delta \phi=\pi)$ excitations with two electrodes located along the wire.

Two resonance peaks similar to those excited with one electrode are observed in the in-phase excitation $(\Delta \phi=$ $0)$. Conversely, no distinguished excitation of the SSW was caused by the opposite-phase excitation $(\Delta \phi=\pi)$. This result indicates that opposite PMA changes at different positions cancel out through the wire for both of the linear and parametric mode excitations, which can be used as phases comparing microwave voltages

Another example of spatial integration of the voltage-induced SSW excitation with two electrodes is demonstrated in Fig. 8. The same device structure, as that shown in Fig. 7, was assumed. When an in-phase PMA modulation with $f_{\mathrm{exc}}=5.44 \mathrm{GHz}$ was applied with two electrodes $\left(\mathrm{G}_{\mathrm{A}}: 1, \mathrm{G}_{\mathrm{B}}: 1\right)$, a parametric $\mathrm{SSW}$ was excited with $\Delta H_{\mathrm{k}}$ larger than 200 Oe. A micromagnetic configuration of the SSW excited with two electrodes is shown in Fig. 8(b). Conversely, an SSW was not formed with a single-electrode excitation $\left(\mathrm{G}_{\mathrm{A}}: 0, \mathrm{G}_{\mathrm{B}}: 1\right)$, even when $\Delta H_{\mathrm{k}}$ was larger than twice the excitation threshold for the two-electrode excitation. Note that the precession amplitude for the single-electrode excitation was suppressed within the thermal noise level due to the existence of a threshold on the parallel parametric excitation. These results should be applicable to the AND gate operation for nanostructured SW logic devices. Noise floor for the $\Delta H_{\mathrm{k}}$ lower than 200 Oe $\left(\mathrm{G}_{\mathrm{A}}: 1, \mathrm{G}_{\mathrm{B}}: 1\right)$ was somewhat smaller than that shown in Fig.7 $(\Delta \phi=0)$, since the higher $\Delta H_{\mathrm{k}}$ of 500 Oe was assumed in the spectra simulations of Fig. 7. The noise level of the single-electrode excitation also exhibits smaller value independent on the value of $\Delta H_{\mathrm{k}}$.

\section{Conclusions}

The voltage-induced excitation of geometrically confined SSWs in nanowires with perpendicular anisotropy was numerically studied via micromagnetic simulations. Sinusoidal modulations of perpendicular anisotropy fields were found to cause linear and parametric mode excitations of SSWs depending on the external field. The dependence of the SSW resonance properties on the structural parameters was systematically examined. A possible application to a SSW-based logic operation was demonstrated in a nanowire with lateral dimensions of $20 \mathrm{~nm} \times 100 \mathrm{~nm}$, where the spatial integration effect of the voltage-induced anisotropy modulation was used.

Acknowledgements This study has been partly supported by the Japan Society for the Promotion of Science (JSPS) KAKENHI under Grand-in-aid Number JP17J09702.

\section{References}
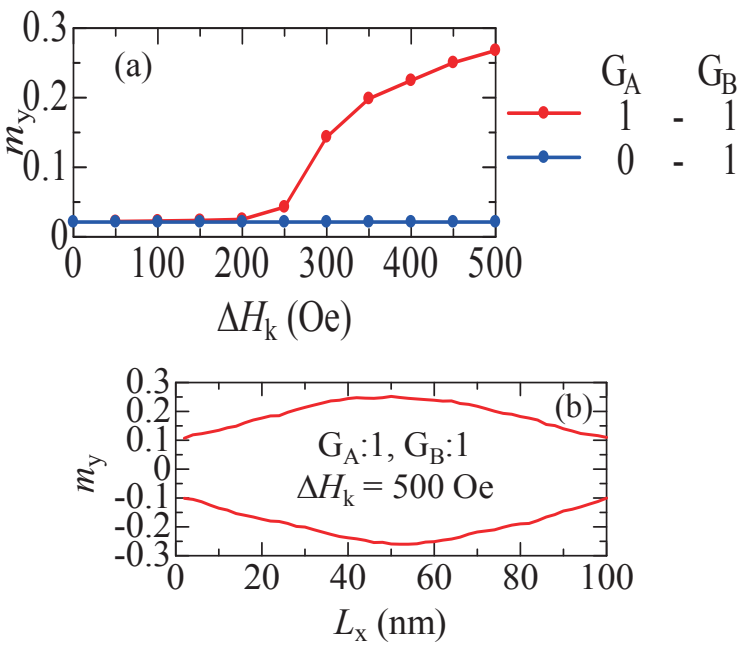

Fig. 8 (a) Comparison between the SSW precession amplitude for the one-gate $\left(\mathrm{G}_{\mathrm{A}}: 0, \mathrm{G}_{\mathrm{B}}: 1\right)$ and simultaneous two-gate $\left(\mathrm{G}_{\mathrm{A}}: 1, \quad \mathrm{G}_{\mathrm{B}}: 1\right)$ excitations. (b) Micromagnetic configuration of the SSW excited with two electrodes.
1) B. Hillebrands and K. Ounadjela, "Spin dynacs in confined magnetic structutrees 1", Springer (2001).

2) G. Bertotti, I. Mayergoyz and C. Serpico, "Nonlinear magnetization dynamics in nanosystems", Ersevier, (2009).

3) H. Zabel and M. Farle (Eds), "Magnetic Nanostructures", Springer Tracts in Modern Physics, 246 (2013).

4) A. G. Gurevich and G. A. Melkov, "Magnetization oscillations and waves", CRC Press (1996).

5) D. D. Stancil and A. Prabhakar, "Spin waves", Springer (2009).

6) V. V. Kruglyyak, S. O. Demokritov and D. Grundler, "Magnonics", J. Phys. D: Appl. Phys.43, 264001 (2010).

7) B. Lenk, H. Ulrichs, F. Garbs and M. Munzenberg, Physics Reports, 507, 107 (2011).

8) A. V. Chumak, V. I. Vasyuchka, A. A. Serga and B. Hillebrands, Nature Phys., 11, 453 (2015).

9) F. Matsukura, Y. Tokura and H. Ohno, Nature Nanotech. 10, 209 (2015).

10) H. Kurebayashi, O. Dzyapko, V. E. Demidov, D. Fang, A. J. Ferguson and S. O. Demokritov, Appl. Phys. Lett., 99, 162502 (2011).

11) C. W. Sandweg, Y. Kajiwara, A. V. Chumak, A. A. Serga, V. I. Vasyuchka, M. B. Jungfleisch, E. Saitoh and B. Hillebrands, Phys. Rev. Latt., 106, 216601 (2011). 
12) A. A. Serga, C. W. Sandweg, V. I. Vasyuchka, M. B. Jungfleish, B. Hillebrands, A. Kreisel, P. Kopietz and M. P. Kostylev, Phys. Rev. B 86, 134403 (2012).

13) G. A. Melkov, Yu. V. Koblyanskiy, R. A. Slipets, A. V. Talalaevskij and A. N. Slavin, Phys. Rev. B 79, 134411 (2009).

14) S. Urazhdin, V. Tiberkevich and A. Slavin, Phys. Rev. Lett., 105, 237204 (2010).

15) H. Ulrichs, V. E. Demidov, S. Demokritov and S. Urazhdin, Phys. Rev. B 84, 094401 (2011).

16) T. Bracher, P. Pirro, B. Obry, B. Leven, A. A. Serga and B. Hillebrands, Appl, Phys. Lett., 99, 162501 (2011).

17) T. Bracher, P. Pirro, A. A. Serga and B. Hillebrands, Appl. Phys. Lett., 103, 142415 (2013).

18) T. Bracher, P. Pirro, F. Heussner, A. A. Serga and B. Hillebrands, Appl. Phys. Lett., 104, 092418 (2014).

19) A. Capua, C. Rettner and S. Parkin, Phys. Rev. Lett., 116, 0247204 (2016).

20) M. Weisheit, S. Fähler, A. Marty, Y. Souche, C. Poinsignon and D. Givord, Science 315, 349 (2007).

21) C-G Duan, J. P. Velev, R. F. Sabirianov, Z. Zhu, J. Chu, S. S. Jaswal and E. Y. Tsymbal, Phys. Rev. Ret. 101, 137201 (2008).

22) D. Chiba, M. Sawicki, Y. Nishitani, Y. Nakatani, F. Matsukura and H. Ohno, Nature 455, 515 (2008).

23) M. Tsujikawa and T. Oda, Phys. Rev. Lett., 102, 247203 (2009).

24) T. Nozaki, Y. Shiota, M. Shiraishi, T. Shinjo and Y. Suzuki, Appl. Phys. Lett., 96, 022506 (2010).

25) T. Nozaki, Y. Shiota, S. Miwa, S. Murakami, F. Bonell, S. Ishibashi, H. Kubota, K. Yakushiji, T. Saruya, A. Fukushima, S. Yuasa, T. Shinjo and Y. Suzuki, Nature Phys. 8, 491 (2012).
26) S. Kanai, M. Gajek, D. C. Worledge, F. Matsukura and H. Ohno, "Electric field-induced ferromagnetic resonance in a $\mathrm{CoFeB} / \mathrm{MgO}$ magnetic tunnel junction under dc bias voltages", Appl Phys. Lett. 195, 242409 (2014).

27) K. Miura, S. Yabuuchi, M. Yamada, M. Ichimura, B. Rana, S. Ogawa H. Takahashi, Y. Fukuma, Y. Otani, "Voltage-induced magnetization dynamics in $\mathrm{CoFeB} /$ $\mathrm{MgO} / \mathrm{CoFeB}$ magnetic tunnel junctions", Scientific Reports 7, 42511 (2017).

28) Y-J. Chen, H. K. Lee, R. Verba, J. A. Katine, I. Barsukov, V. Tiberkevich, J. Q. Xiao, A. N. Slavin and I. N. Krivorotov, "Parametric Resonance of Magnetization Excited by Electric Field", Nano Lett. 17, 572 (2017).

29) R. Verba, V. Tiberkevich, I. Krivorotov and Andrei Slavin, Phys. Rev. Appl. 1, 044006 (2014).

30) R. Verba, M. Carpentieri, G. Finocchio, V. Tiberkevich and A. Slavin, Scientific Reports 6, 25018 (2016),

31) Y. Shiota, F. Bonell, S. Miwa, N. Mizuochi, T. Shinjo and Yoshishige Suzuki, Appl. Phys. Lett., 103, 082410 (2013).

32) Y. Shiota, T. Nozaki, S. Tamaru, K. Yakushiji, H. Kubota, A. Fukushima, S. Yuasa and Y. Suzuki, Appl. Phys. Lett., 111, 022408 (2017).

33) W. F. Brown, Phys. Rev, 130, 1677 (1963).

34) O. Chubykalo, J. D. Hannay, M. Wongsam, R. W. Chantrell and J. M. Gonzalez, Phys. Rev. B. 65, 184428 (2002).

35) V. D. Tsiantos, D. Suess, T. Schrefl and J. Fidler, J. Appl. Phys., 89, 7600 (2001).

Received Oct. 08, 2019; Accepted Jan. 07. 2020 